\title{
Avaliação de HPAs nos manguezais dos Estuários dos Rios São Paulo e Jaguaripe, Bahia de Todos os Santos, Bahia
}

\section{Liliane Santos de Araújo1,*, Paulo Oliveira Mafalda Jr. $^{2}$, Ícaro Thiago Andrade Moreira ${ }^{1}$}

${ }^{1}$ Universidade Federal da Bahia. Departamento de Geoquímica. Laboratório de Estudo de Petróleo. Salvador-BA, Brasil (CEP 40170-020). *E-mail: lilyuefs@yahoo.com.br.

${ }^{2}$ Universidade Federal da Bahia. Departamento de Biologia. Laboratório de Plâncton. Salvador-BA, Brasil (CEP 40170-115).

Resumo. Os manguezais são importantes ecossistemas para a proteção da linha costeira, abrigo para animais residentes e imigrantes, e ciclagem de nutrientes. No entanto, ações antrópicas como a queima de combustíveis fósseis resulta na liberação de contaminantes para o receptor hídrico, como os Hidrocarbonetos Policíclicos Aromáticos (HPAs), que possuem características tóxicas e carcinogênicas. Desse modo, o presente estudo tem como objetivo avaliar os HPAs nos sedimentos do Estuário do Rio São Paulo e do Estuário do Rio Jaguaripe, assim como determinar a origem da contaminação. Para isto, foi realizada duas coletas em cada estuário, no período chuvoso, em agosto de 2015, e no período seco, em dezembro de 2015, em cinco pontos distribuídos ao longo de cada manguezal, sendo coletadas duas amostras por ponto, totalizando 10 unidades amostrais. 0 Estuário do Rio São Paulo localiza-se ao norte da Baía de Todos os Santos (BTS), no Município de Candeias, nas proximidades de indústrias químicas e petroquímicas, e o Estuário do Rio Jaguaripe localiza-se no Município de Nazaré, no sudoeste da BTS, uma área visivelmente menos impactada. A concentração total dos HPAs analisados variou de 3,38 ng.g ${ }^{-1}$ a 189,02 ng.g ${ }^{-1}$, no Estuário do Rio Jaguaripe, e de 3,00 ng.g ${ }^{-1}$ a 128,63 ng.g- ${ }^{-1}$, no Estuário do Rio São Paulo, de acordo com o CONAMA não apresentam riscos de contaminação nos organismos marinhos presentes nestes locais.

Palavras-chave: Origem de HPAs; Níveis de contaminação; Sedimento.

Abstract. Evaluation of HPAs in the mangroves of the Estuaries of the São Paulo and Jaguaripe Rivers, Baía de Todos os Santos, Bahia. The Mangroves are important ecosystems for protecting the shoreline, shelter for residents and immigrants, and for nutrient cycling. However, anthropogenic actions such as burning fossils result in the release of contaminants to the water
Recebido:

$13 / 04 / 2018$

Aceito:

20/07/2018

Publicado:

$31 / 08 / 2018$

Acesso aberto

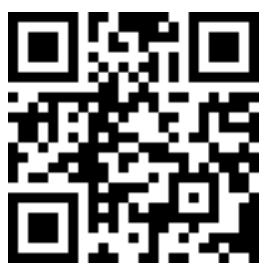

ORCID

(1) 0000-0002-6724-0610

Liliane Santos de

Araújo

(D) 0000-0002-5542-3671

Paulo Oliveira Mafalda Jr.

D 0000-0002-3964-7368 Ícaro Thiago Andrade Moreira 
receptor, such as PAHs, which have carcinogenic and toxic characteristics. Thus, the present study aims to evaluate the sedimentary PAHs of the Estuarie of the River São Paulo and Estuarie of River Jaguaripe, as well as to determine the origin of the contamination. For this purpose, two collections were carried out in each estuary in the rainy season in August 2015 and in the dry period in December 2015 at five points throughout the mangrove, being collected two samples each point, totalling ten sample units. The São Paulo Estuary is located in the north of the Baía de Todos os Santos (BTS), in Candeias and surrounding petrochemical areas, and the Estuary of the Jaguaripe River is located in the Municipality of Nazaré, Southwest of BTS, a visibly less impacted area. The total HPA concentration ranged from 3.38 to $189.02 \mathrm{ng} . \mathrm{g}^{-1}$ in Jaguaripe and from 3.00 to $128.63 \mathrm{ng}$ g-1 in the São Paulo Estuary that according to CONAMA does not present contamination risks in the marine organisms present in these locations.

Keywords: Source of PAHs; Levels of contamination; Sediment.

\section{Introdução}

Os Hidrocarbonetos Policíclicos Aromáticos (HPAs) são compostos que possuem dois ou mais anéis aromáticos condensados, são persistentes no ambiente e possuem baixa solubilidade em água e características lipofílicas, que tendem a acumular em estruturas corpóreas de organismos marinhos e nas folhas das plantas (Caruso e Alaburda, 2008). Devido à sua característica hidrofóbica os HPAs são adsorvidos pelo material particulado e armazenado no sedimento (Wise et al., 1995; Caruso e Alaburda, 2008).

Deste modo, alguns animais filtradores, como ostras e mexilhões, ou animais que permanecem em contato com o sedimento com alto teor de HPAs, acabam acumulando estes compostos no organismo. Nos seres humanos, estes compostos são metabolizados para posterior excreção na urina, que podem reagir com a guanina do DNA e resultar em processos de tumoração (Caruso e Alaburda, 2008).

Os HPAs são provenientes de processos naturais, como erupções vulcânicas e queimadas, ou de ações antropogênicas, resultantes da combustão incompleta da matéria orgânica, de produtos derivados do petróleo, que atingem o ambiente marinho por meio de efluentes domésticos e industriais, escapamento de veículos, drenagem urbana ou deposição atmosférica (Witt, 1995; Le Bihanic et al., 2014).

A Baía de Todos os Santos (BTS) está localizada no estado da Bahia, que se estende por $462 \mathrm{Km}$ de costa, importante para o país devido a sua extensão, sendo considerada como maior e mais importante baía navegável da costa do Brasil, sendo drenada pelos Rios Paraguaçu, Jaguaripe e Subaé (Onofre et al., 2007; Hadlich et al., 2009).

A relevância deste trabalho baseia-se na possível indicação de contaminação por HPAs nos manguezais do Estuário do Rio São Paulo, resultante de sua proximidade em relação à Região Metropolitana de Salvador e de atividades industriais químicas $\mathrm{e}$ petroquímicas, e do Estuário do Rio Jaguaripe, que se localiza nas proximidades da rodovia BA 001 e que apresenta ao longo do seu corpo vários efluentes domésticos.

Desta forma, o presente estudo tem como objetivo geral a avaliação da concentração de HPAs no sedimento do 
Estuário do Rio São Paulo e do Estuário do Rio Jaguaripe, além de indicar sua possível fonte de contaminação, podendo contribuir para discussões sobre 0 estado de conservação desta área costeira.

\section{Materiais e métodos}

\section{Área de estudo}

0 presente trabalho apresenta como áreas de estudo do Estuário do Rio São Paulo e do Estuário do Rio Jaguaripe (Figura 1).

\section{Estuário do Rio São Paulo}

Estuário do Rio São Paulo, está localizado na Baía de Todos os Santos (BTS), no Município de Candeias, a aproximadamente 46,1 km de Salvador, capital do Estado da Bahia, que o coloca como parte integrante da área metropolitana. Possui aproximadamente $1.100 \mathrm{~km}^{2}$ e apresenta $32 \mathrm{~km}$ de largura. Este município tem como áreas limite o Município de São Francisco do Conde, a oeste, Simões Filho, a sudeste, São Sebastião do Passé, ao norte, e Salvador, ao sul (Bahia, 2004). Possui em seu entorno indústrias petrolíferas $\mathrm{e}$ efluentes domésticos em seu corpo hídrico.

Possui o manguezal como o principal ecossistema, que serve como fonte de renda, lazer e economia para a população local.

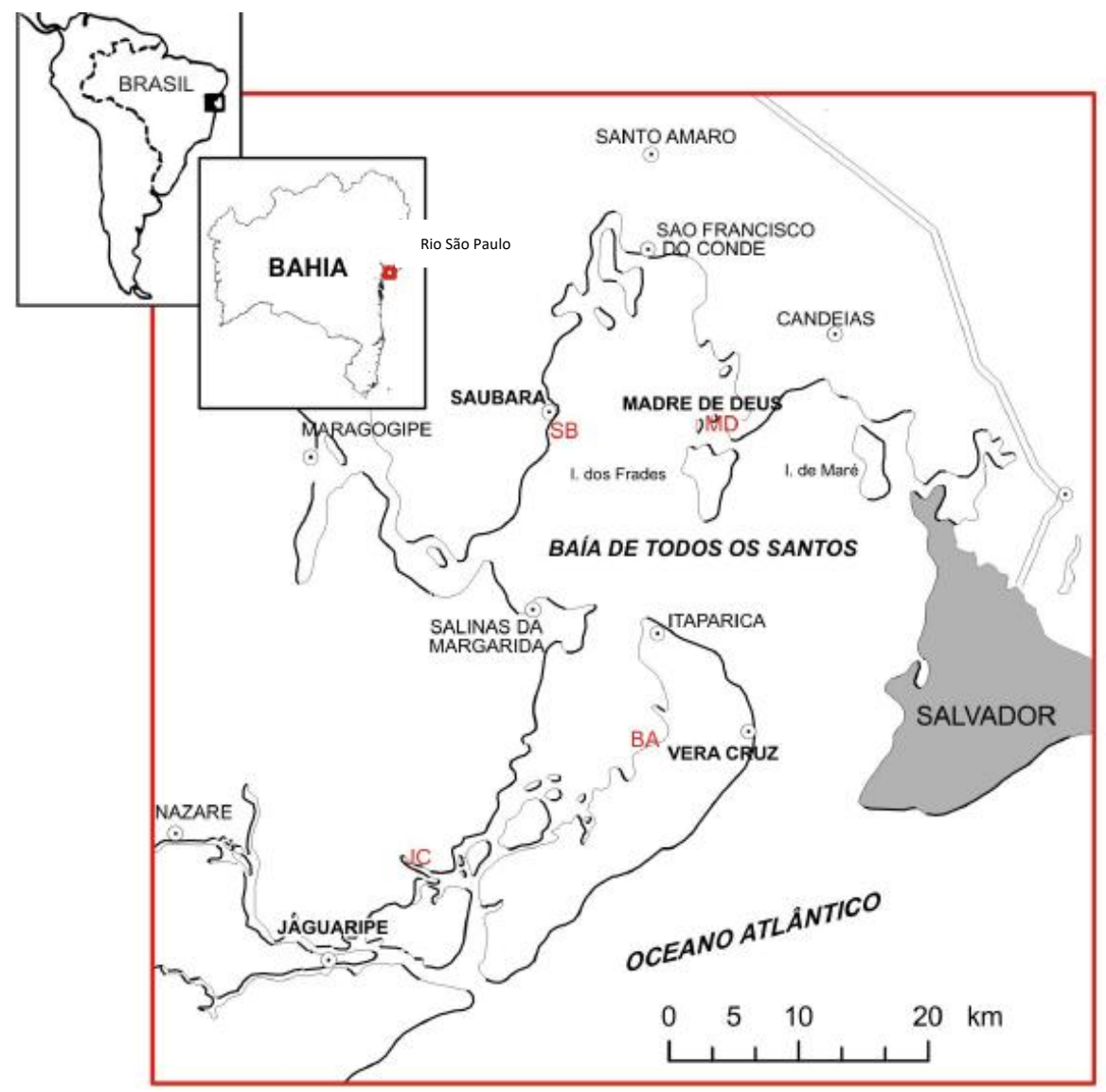

Figura 1. Mapa da Baia de Todos os Santos (BTS) e regiões de amostragem, Rio Jaguaripe e Rio São Paulo. Fonte: Bahia (2004). 


\section{Estuário do Rio Jaguaripe}

O Estuário do Rio Jaguaripe (Figura 1) está localizado na região sudoeste da Baía de Todos os Santos, próximo ao município de Nazaré. Possui uma extensão de aproximadamente $4 \mathrm{~km}$ de deltas de maré (Bittencourt et al., 2001).

A agricultura apresenta-se como atividade principal em seu entorno o que gera impacto ambiental nesta área resultante do uso de pesticidas, assim como o aterro do manguezal, efluentes domésticos e resíduos sólidos.

\section{Coleta do sedimento}

As amostras de sedimentos foram coletadas a $1 \mathrm{~m}$ de profundidade, com auxílio de um testemunho, em cinco pontos ao longo do manguezal do Rio São Paulo e do Rio Jaguaripe, totalizando 10 unidades amostrais, em casa manguezal. Após coletadas as amostras, foram armazenadas em recipientes de vidro devidamente identificados e previamente descontaminados e mantidos a uma temperatura aproximada de $4{ }^{\circ} \mathrm{C}$ antes de serem encaminhados ao Laboratório de Estudo de Petróleo (LEPETRO), da Universidade Federal da Bahia (UFBA).

\section{Tratamento do material}

Foram utilizados na composição do processo analítico, sulfato de sódio e sílica, ambos da marca Merck, que foram colocados em forno mufla, durante $4 \mathrm{~h}$ a uma temperatura de $450{ }^{\circ} \mathrm{C}$, para remover possíveis resíduos interferentes, ativando dessa forma a sílica. Em seguida, foram armazenados em recipiente de vidro tampado e postos em dessecador.

\section{sedimento \\ Determinação de HPAs no}

A determinação de HPAs nas amostras de sedimentos foi baseada no método EPA $3540 \mathrm{C}$ (EPA, 1996). Alíquotas de $10 \mathrm{~g}$ de sedimento foi utilizada para a extração Soxlhet por $4 \mathrm{~h}$, utilizado-se $150 \mathrm{~mL}$ de diclorometano como solvente, após a adição do padrão surrogate, p-terphenyl d-14, para avaliar a taxa de recuperação. 0 extrato foi evaporado em evaporador rotativo a 1 $\mathrm{mL}$ e posteriormente feito a etapa de clean-up para remover possíveis interferentes no processo posterior de leitura dos HPAs.

\section{extratos}

\section{Etapa de purificação dos}

Foi utilizada coluna cromatográfica de vidro, empacotada com $10 \mathrm{~g}$ de sílica e uma fina camada de sulfato de sódio anidro, em seguida foi adicionado uma alíquota de $1 \mathrm{~mL}$ do extrato de sedimento e acrescentado $50 \mathrm{~mL} \mathrm{(1:1)} \mathrm{da}$ mistura diclorometano e hexano como eluente. Em seguida, o resultante foi concentrado em $1 \mathrm{~mL}$ no rotovapor, posto em vial e armazenado em freezer a $-18^{\circ} \mathrm{C}$ até $\mathrm{o}$ momento da análise.

\section{HPAs}

\section{Identificação das origens dos}

Foram utilizadas três razões diagnósticas para indicar as possíveis fontes dos HPAs encontrados nos sedimentos do Estuário do Rio São Paulo e no Estuário do Rio Jaguaripe: Fluoranteno/Pireno (FLA/PIR); benzo(a) antraceno/(benzo(a) antraceno + criseno) $(\mathrm{BaA} /(\mathrm{BaA}+\mathrm{Cri})$ e o somatório dos HPAs de alta massa molecular/soma dos HPAs de baixa massa molecular ( $\Sigma$ LMW/ $\Sigma$ HMW).

\section{Resultados e discussão}

De acordo com a Resolução CONAMA no 454/2012 (Brasil, 2012) as concentrações dos HPAs são classificados no nível 1 , que apresenta limiar abaixo do qual há menor probabilidade de efeitos adversos à biota, e nível 2, que apresenta limiar acima do qual há maior probabilidade de efeitos adversos à biota. A Tabela 1 consta os valores estabelecidos pelo Conselho Nacional de Meio Ambiente (CONAMA) e os valores encontrados nos sedimentos do Estuário 
do Rio São Paulo e do Estuário do Rio

Jaguaripe, no período chuvoso e seco.

Tabela 1. Diretrizes de qualidade de sedimento (concentrações em ng.g-1), segundo a Resolução CONAMA no 454/2012, em água salobra no Estuário do Rio São Paulo e no Estuário do Rio Jaguaripe.

\begin{tabular}{lcccccc}
\hline \multirow{2}{*}{ HPAs } & Nível 1 & Nível 2 & Jag. 1 & Jag. 2 & SP1 & SP2 \\
\cline { 2 - 6 } & AS & AS & PC & PS & PC & PS \\
\hline NAF & 160 & 2.100 & 30,56 & $\mathbf{6 7 , 6 4}$ & 27,09 & $\mathbf{4 3 , 3 5}$ \\
AcNf & 44 & 640 & 12,95 & 14,97 & 23,33 & 23,85 \\
Can & 16 & 500 & 3,38 & 3,96 & 1,24 & 3,00 \\
Fl & 19 & 540 & 8,50 & 11,11 & 5,43 & 9,55 \\
Fen & 240 & 1.500 & 45,01 & $\mathbf{5 2 , 0 4}$ & 12,86 & 28,45 \\
A.n & 85,3 & 1.100 & 16,98 & 14,66 & 19,97 & 25,92 \\
Flu & 600 & 5.100 & 128,64 & $\mathbf{1 8 9 , 0 2}$ & 35,69 & $\mathbf{5 3 , 1 9}$ \\
Pir & 665 & 2.600 & 77,99 & $\mathbf{9 6 , 2 6}$ & 32,31 & 61,24 \\
BaA & 280 & 690 & 50,25 & 47,90 & 13,29 & 30,06 \\
Cri & 300 & 850 & 48,84 & 37,85 & 11,24 & 34,17 \\
BbFla & - & - & 97,32 & $\mathbf{9 8 , 8 4}$ & 64,57 & $\mathbf{1 0 3 , 6 8}$ \\
BkFla & - & - & 44,90 & 36,71 & 17,78 & 29,46 \\
BaP & 230 & 760 & 77,62 & 71,86 & 31,78 & 55,59 \\
IP & - & - & 41,08 & $\mathbf{9 0 , 7 3}$ & 46,41 & $\mathbf{8 0 , 4 8}$ \\
DBahA & 43 & 140 & 20,84 & 21,27 & 19,06 & 29,79 \\
BghiP & - & - & 42,46 & $\mathbf{8 5 , 2 1}$ & 55,54 & $\mathbf{9 0 , 7 5}$ \\
EHPAs & 4.000 & - & 642,750 & 938,36 & 423,90 & 751,24 \\
\hline
\end{tabular}

Legenda: $\mathrm{AS}=$ água salobra, $\mathrm{PC}=$ período chuvoso, $\mathrm{PS}=$ período seco, Jag. = Jaguaripe, $\mathrm{SP}=\mathrm{São}$ Paulo.

Dentre os 16 HPAs encontrados nos sedimentos do Estuário do Rio São Paulo e do Estuário do Rio Jaguaripe, os compostos naftaleno, fluoranteno, pireno, benzo(b) fluoranteno, e benzo (gui) perileno apresentaram maiores concentrações, que, segundo a International Agency for Research on Cancer (IARC, 2010), são classificados como não carcinogênicos, com exceção do composto benzo (b) fluoranteno que é fortemente carcinogênico.

No entanto apresentaram valores inferiores ao nível 1 , que determina limiar abaixo do qual a menor probabilidade de efeitos adversos a biota. Este resultado difere dos valores encontrados por Silva et al. (2014) na BTS, onde obteve alto, médio e baixo níveis de concentrações dos HPAs nos sedimentos.

\section{Origem dos HPAs}

Índices diagnósticos são utilizados para determinar a origem dos HPAs que podem ser de fonte antropogênica ou natural, sendo as de origem antropogênica, pirolítica e petrogênica, de maior ocorrência nas amostras ambientais (Caruso e Alaburda, 2008; He et al., 2014), que estão frequentemente presentes nos HPAs de alta massa molecular, enquanto os HPAs de baixa massa molecular são encontrados principalmente nos hidrocarbonetos do petróleo (Notar, 1999; Silva et al., 2014).

Deste modo, foram utilizadas três razões diagnósticas para indicar as possíveis fontes dos HPAs encontrados nos sedimentos do Estuário do Rio São Paulo e do Estuário do Rio Jaguaripe. Fenantreno/antraceno (FEN/ANT), Fluoranteno/Pireno (FLA/PIR) e o somatório dos HPAs de alta massa molecular/soma dos HPAs de baixa massa molecular ( $\Sigma$ LMW $/ \Sigma$ HMW).

A relação FEN/ANT maior que 10 tem sua contaminação resultante principalmente de fontes petrogênicas e valores abaixo indicam contaminação de origem pirogênica (Budzinski et al., 1997). O resultado do parâmetro geoquímico FLU/PIR maior que 1 indica 
fonte pirogênica e menores que 1 indica fonte petrogênica (Yancheshmeh et al., 2014), enquanto os valores obtidos da razão $\Sigma$ LMW/ $\Sigma$ HMW menores que 1 indicam contaminação pirogênica, valores maiores que 1 , apontam fonte petrogênica (Soclo et al., 2000).

A Tabela 2 mostra as razões analisadas $\mathrm{e}$ as possíveis fontes de origem dos HPAs nos dois estuários estudados. Pode-se observar que no Estuário do Rio Jaguaripe nas três razões diagnósticas utilizadas predominou a origem pirogênica, que é caracterizada pela combustão incompleta da matéria orgânica, com valores médios e desvio padrão variando de 0,21 ng.g $^{-1} \pm 0,02$ ng.g ${ }^{-1}$ a 3,61 ng.g ${ }^{-1} \pm 0,99$ ng..$^{-1}$, respectivamente. A origem pirogênica neste local é oriunda da proximidade com a BA 001 que devido à ação pluviométrica arrastam os resíduos da queima de combustíveis fósseis, como gasolina e óleo diesel, proveniente dos automóveis para o corpo hídrico do manguezal, além dos efluentes domésticos presentes no manguezal.

Já no Estuário do Rio São Paulo, apenas a razão diagnóstica FLA/PYR indicou origem petrogênica, no período seco, com valores médios e desvio padrão de 0,73 ng.g $^{-1} \pm 0,30$ ng.g ${ }^{-1}$, enquanto as razões FEN/ANT e

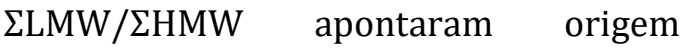
pirogênica. Portanto, considera-se a fonte contaminante de HPAs neste local de origem pirogênica, resultante de efluentes domésticos e industriais presentes ao longo do manguezal.

Tabela 2. Parâmetros diagnósticos de origem de HPAs no Estuário do Rio São Paulo e no Estuário do Rio Jaguaripe.

\begin{tabular}{cccccc}
\hline Período & Razão & Limite & Origem & JP- Média e DP & SP- Média e DP \\
\hline PC & \multirow{2}{*}{ FEN/ANT } & $>10$ & Petrogênica & $0,51 \pm 0,09$ & $0,73 \pm 0,35$ \\
PS & & $<10$ & Pirogênica & $3,61 \pm 0,99$ & $1,14 \pm 0,78$ \\
PC & \multirow{2}{*}{ FLA/PYR } & $>1$ & Pirogênica & $1,70 \pm 0,41$ & $1,05 \pm 0,24$ \\
PS & & $<1$ & Petrogênica & $1,98 \pm 0,46$ & $0,73 \pm 0,30$ \\
PC & \multirow{2}{*}{ LMWW/SHMW } & $<1$ & Pirogênica & $0,21 \pm 0,02$ & $0,32 \pm 0,13$ \\
PS & $>1$ & Petrogênica & $0,22 \pm 0,04$ & $0,39 \pm 0,21$ \\
\hline
\end{tabular}

Legenda: $\quad \mathrm{PC}=$ Período chuvoso, $\quad \mathrm{PS}=$ período seco, Fen $=$ fenantreno, $\quad$ Ant $=$ antraceno, $\mathrm{Fla}=$ fluoranteno, $\mathrm{Pyr}=$ pireno, $\Sigma \mathrm{LMW}=$ soma dos HPAs de baixa massa molecular, $\Sigma \mathrm{HMW}=$ soma dos HPAs de alta massa molecular

\section{Conclusão}

De acordo com a Resolução CONAMA no 454/2012 (Brasil, 2012) pode-se concluir que os sedimentos dos estuários dos rios São Paulo e Jaguaripe encontram-se em baixas concentrações que indica que não há contaminações por HPAs nas áreas analisadas. É importante salientar que o sedimento foi coletado a 1 $m$ de profundidade em ambos os estuários devido à permanência de organismos de fonte alimentícia e de renda nesta profundidade por quase todo o ano.

Os resultados obtidos das três razões diagnósticas para verificar a origem dos HPAs foram similares, apontando para uma fonte pirolítica, possivelmente oriundos de efluentes domésticos e indústrias presentes em ambos os estuários estudados, ou da proximidade com estradas em que o fluxo de automóveis é intenso.

Deste modo, faz-se necessário o monitoramento nestes estuários visando à conservação ambiental por meio de 
ações preventivas e correta destinação dos efluentes, já que a BTS é uma área de importantes atividades como turismo, lazer e mariscagem.

\section{Conflito de interesses}

Os autores declaram não haver conflito de interesses.

\section{Referências}

Bahia. Diagnóstico do grau de contaminação da Baía de Todos os Santos por metais pesados e hidrocarbonetos de petróleo a partir da análise das suas concentrações nos sedimentos de fundo e biota associada. Salvador: CRA, 2004. (Relatório Técnico).

Bittencourt, A. C.S.P.; Lessa, G. C.; Dominguez, J. M. L.; Martin, L.; Vilas-Boas, G. S.; Farias, F. F. High and low frequency erosive and constructive cycles in estuarine beaches: an example from Garcez Point, Bahia/Brazil. Anais da Academia Brasileira de Ciências, v. 73, n. 4, p. 599-610, 2001. https://doi.org/10.1590/S0001-3765200 1000400013

Brasil. Resolução CONAMA no 454, de 1 de novembro de 2012. Estabelece as diretrizes gerais e os procedimentos referenciais para o gerenciamento do material a ser dragado em águas sob jurisdição nacional. Disponível em: <http://www.mma.gov.br/port/conama/legi abre.cfm?codlegi=693>. Acesso em: 01 mar. 2016.

Budzinski, H.; Jones, I.; Bellocq, J.; Piérard, C.; Garrigues, P. Evaluation of sediment contamination by polycyclic aromatic hydrocarbons in the Gironde Estuary. Marine Chemistry, v. 58 , n. $1 / 2$, p. $85-97$, 1997. https://doi.org/10.1016/S0304-4203 (97)00028-5

Caruso, M. S. F.; Alaburda, J. Hidrocarbonetos Policíclicos Aromáticos benzo(a)pireno: uma revisão. Revista do Instituto Adolfo Lutz, v. 67, $\mathrm{n}^{\circ} 1$, p. 1-27, 2008. Disponível em: <http://revistas.bvs-vet.org.br/rialutz/ article/view/7180/7405>. Acesso em: 01 mar. 2016.

EPA-United States Environmental Protection Agency. Method 3540 C. Soxhlet Extraction. Washington: EPA, 1996. Disponível em: <https://www.epa.gov/sites/ production/files/2015-12/documents/ 3540c.pdf>. Acesso em: 01 mar. 2016.
Hadlich, G. M.; Ucha, J. M. Apicuns: aspectos gerais, evolução recente e mudanças climáticas globais. Revista Brasileira de Geomorfologia, v. 10, n. 2, p. 13-20, 2009. https://doi.org/10.20502/rbg.v10i2.126

He, X.; Pang, Y.; Song, X.; Chen, B.; Feng, Z.; $\mathrm{Ma}, \mathrm{Y}$. Distribution, sources and ecological risk assessment of PAHs in surface sediments from Guan River Estuary, China. Marine Pollution Bulletin, v. 80 , n. $1 / 2$, p. 52-58, 2014. https://doi.org/10.1016/j.marpolbul. 2014.01.051

IARC - International Agency for Research on Cancer. Working Group on the Evaluation of Carcinogenic Risks to Humans. Some nonheterocyclic polycyclic aromatic hydrocarbons and some related exposures. IARC, 2010. (IARC monographs on the Evaluation of Carcinogenic Risks to Humans/World Health Organization, International Agency for Research on Cancer).

Le Bihanic, F.; Morin, B.; Cousin, X.; Le Menach, K.; Budzinski, H.; Cachot, J. Developmental toxicity of PAH mixtures in fish early life stages. Part I: adverse effects in rainbow trout. Environmental Science and Pollution Research, v. 21, n. 24, p. 13720 13731, 2014. https://doi.org/10.1007/ s11356-014-2804-0

Notar, M.; Leskovšek, H.; Faganeli, J. Composition, distribution and sources of Polycyclic Aromatic Hydrocarbons in sediments of the Gulf of Trieste, Northern Adriatic Sea. Marine Pollution Bulletin, v. 42, n. 1, p. 36-44, 1999. https://doi.org/ 10.1016/S0025-326X(00)00092-8

Onofre, C. R. E.; Celino, J. J.; Nano, R. M. W.; Queiroz, A. F. S. Biodisponibilidade de metais traço nos sedimentos de manguezais da porção norte da Baía de Todos os Santos, Bahia, Brasil. Revista de Biologia e Ciências da Terra, v. 7, n. 2, p. 65-82, 2007.

Silva, S. M. T.; Beretta, M.; Tavares, T. M. Diagnóstico da contaminação por Hidrocarbonetos Policíclicos Aromáticos nos sedimentos de mesolitoral da Baía de Todos os Santos, Brasil. Revista Eletrônica de Gestão e Tecnologias Ambientais, v. 2, n. 2, p. 193-204, 2014. https://doi.org/10.17565/ gesta.v2i2.10449

Soclo, H. H.; Garrigues, Ph.; Ewald, M. Origin of polycyclic aromatic hydrocarbons (PAHs) in coastal marine sediments: Case studies in Cotonou (Benin) and Aquitaine (France) 
areas. Marine Pollution Bulletin, v. 40, n. 5, p. 387-396, 2000. https://doi.org/10.1016/ S0025-326X(99)00200-3

Wise, S. A.; Schantz, M. M.; Benner Jr, B. A.; Hays, M. J.; Schiller, S. B. Certification of Polycyclic Aromatic Hydrocarbons in a Marine Sediment Standard Reference Material. Analytical Chemistry, v. 67, n. 7, p.1171-1178, 1995. https://doi.org/ 10.1021/ac00103a006

Witt, G. Polycyclic aromatic hydrocarbons in water and sediment of the Baltic Sea. Marine Pollution Bulletin, v. 31, n. 4/12, p. 237-248, 1995. https://doi.org/10.1016/0025-326X (95)00174-L

Yancheshmeh, R. A.; Bakhtiari, A. R.; Mortazavi, S.; Savabieasfahani, M. Sediment PAH: Contrasting levels in the Caspian Sea and Anzali Wetland. Marine Pollution Bulletin, v. 84, p. 391-400, 2014. https://doi.org/10.1016/j.marpolbul.2014.0 5.001

Informação da Licença: Este é um artigo Open Access distribuído sob os termos da Licença Creative Commons Attribution, que permite uso irrestrito, distribuição e reprodução em qualquer meio, desde que a obra original seja devidamente citada. 